\title{
Prophylactic and Therapeutic Role of Vitamin D Supplementation in COVID-19: A Review
}

\author{
Mehrie Harshad Patel, Sakshi Mishra, Ketul S. Barot, Saloni H. Naik, Angelina Browne, Zara Ahmed, \\ Hemalatha Tumkur Ranganathan, Simmy Lahori, Vraj S. Patel, Siddharth Gupta, Ghulam Shahar Bano, \\ Hassaan Shaikh, Kinal Paresh Bhatt, Muhammad Jamal, Miguel Diaz, John Halpern, \\ Vladimir Derenoncourt, Natalie Leroy, Douglas Gotlin, and Luis Del Prado
}

\section{ABSTRACT}

Looking at the current scenario of the global pandemic with Coronavirus Disease 2019 (COVID-19) and the dramatic rise of variant COVID-19 cases it is reasonable to reason that one's immunity (innate, adaptive, or passive) plays a vital role in an individual's susceptibility as well as the severity. While the population is getting passively immunized with vaccination, the innate and adaptive immune response should also be emphasized. Vitamins and minerals play an important role in developing and modulating the immune response in the human body. Of which, one is, Vitamin D. Besides playing a major role in Calcium metabolism, Vitamin D is involved in modulating various immune system pathways to contain the virus, which includes dampening Severe Acute Respiratory Syndrome Coronavirus 2 (SARS-CoV2) entry and replication, reducing pro-inflammatory cytokines and increasing anti-inflammatory cytokines, increasing the synthesis of a natural antimicrobial peptide, and activating protective cells such as macrophages that can kill SARS-CoV-2. Vitamin D also possesses a neuroprotective property that is linked to the modulation of neurotrophins. In the current situation of the pandemic, an important connection between Vitamin D deficiency and poor health outcomes in COVID-19 patients have been reported. Furthermore, adequate Vitamin D levels were linked to a lower risk of unconsciousness and hypoxia. Vitamin D deficiency affects over half of the world's population. A low serum concentration of Vitamin D is a risk factor for acute respiratory tract infection. Vitamin D supplementation, on the other hand, is linked to a reduced risk of acute respiratory tract infections. Here, we conducted a literature review of publicly available information to summarize the link between Vitamin D levels and the severity of COVID-19 infection. The main objective of this manuscript is to review and provide information reported by published literature from publically available papers on online databases regarding the significance of Vitamin $D$ in immune response and its prophylactic and therapeutic role in the management of COVID-19.

Keywords: Coronavirus, COVID-19, SARS-CoV-2, Vitamin D.
Submitted : June 22, 2021

Published : July 27, 2021

ISSN: $2593-8339$

DOI: 10.24018 /ejmed.2021.3.4.943

Dr. Mehrie H. Patel*

Division of Clinical \& Translational Research, Larkin Health System, South Miami, FL, USA.

(e-mail: patelmehrie@gmail.com) Dr. Sakshi Mishra

Division of Clinical \& Translational Research, Larkin Health System, South Miami, FL, USA.

(e-mail: drmishra.sakshi@gmail.com) Dr. Ketul S. Barot

Pramukhswami Medical College, Anand, Gujarat, India.

(e-mail: ketul.scientist ${ }^{@}$ gmail.com)

Dr. Saloni H. Naik

Pramukhswami Medical College, Anand, Gujarat, India.

(e-mail: nsaloni98@gmail.com)

Dr. Angelina Browne

Division of Clinical \& Translational Research, Larkin Health System, South Miami, FL, USA.

(e-mail: browne.angelina1 @ gmail.com) Dr. Zara Ahmed

Shaheed Zulfiqar Ali Bhutto Medical University (SZABMU), RIHS, Islamabad, Pakistan.

(e-mail: zaraahmed.2395@gmail.com) Dr. Hemalatha Tumkur Ranganathan

Mandya Institute of Medical Sciences, Mandya, Karnataka, India.

(e-mail: Hemalatha.t.ranganathan@ gmail.com)

Dr. Simmy Lahori

Division of Clinical \& Translational Research, Larkin Health System, South Miami, FL, USA.

(e-mail: simmylahori23@gmail.com)

Dr. Vraj S. Patel

Division of Clinical \& Translational Research, Larkin Health System, South Miami, FL, USA.

(e-mail: vrajpate1123@gmail.com) 


\section{INTRODUCTION}

Despite being surrounded by harmful microorganisms, toxins, and the threat of human cells transforming into tumour cells, we manage to survive. This is primarily due to the immune system. The immune system comprises organs, tissues, cells, and molecules that all work together to generate an immune response that protects the body from microorganisms, removes toxins, and destroys tumour cells. The immune system can identify the pathogen, mount an immune response, eliminate the pathogen, and remember it for a future encounter. The immune system can be categorized into three categories: innate immunity (rapid response), adaptive

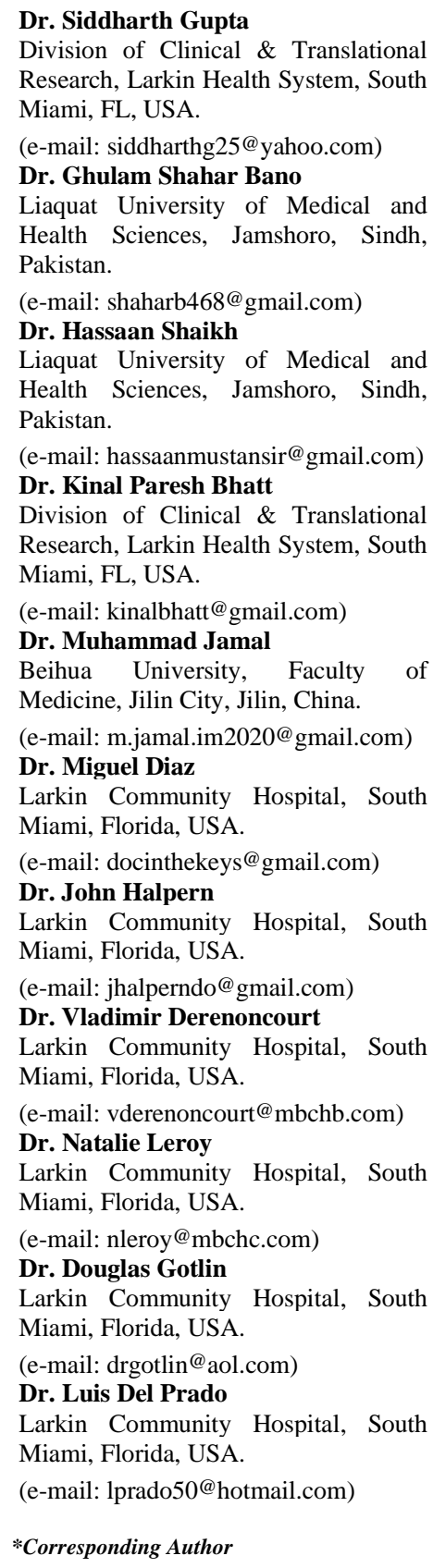

immunity (slow response), and passive immunity. Passive immunity can be natural in the form of antibodies acquired from the mother and artificial in the form of vaccination [1]. A complete immune response consists of initial activation of an innate immune response, followed by activation of the adaptive immune response [1].

The medical fraternity is not unfamiliar with SARS (Severe Acute Respiratory Syndrome) outbreaks. However, at the end of 2019, there was an emerging illness which has come to be known as Coronavirus Disease 2019 (COVID-19), caused by a novel Coronavirus, SARS-CoV-2 (Severe Acute Respiratory Syndrome Coronavirus 2) [2]. This was the beginning of the impending pandemic of the newly emerging disease, one of 
humankind's greatest healthcare challenges. The main route of entry and transmission of SARS-CoV2 is respiratory droplets that are expelled and absorbed by the mucous membranes, particularly the nasal and laryngeal mucosa. The epithelial cells lining these surfaces form a component of innate immunity [3].

Angiotensin-Converting Enzyme-2 (ACE-2) is the main receptor for SARS-Cov2. ACE-2 is present in abundance in some organs, like lung epithelial cells, heart, kidney, gastrointestinal tract, liver, and bladder. Therefore, these organs constitute the main target for this virus. The spike proteins in the virus's envelope bind to the ACE-2 on the host cell surface [4]. Previous animal studies on the SARS CoV suggests a similar mechanism in SARS CoV-2, in which viral spike protein binding is sensed by the essential Toll-like receptor-7 (TLR-7) present on the monocytes, macrophages, and dendritic cells, which then leads to the secretion of alphainterferon, TNF-alpha, and the secretion of interleukin (IL)-12 and IL-6; via various signaling pathways [4].

Along with these events, antigen presentation subsequently results in the formation of CD8+ specific cytotoxic T-cells and, through the CD4+ helper T-cell, leads to the formation of antigen-specific B-cells and antibody production. This thus results in activation of the body's specific adaptive immunity (both humoral and cellular immunity). Additionally, Th17 cells, neutrophils, and granulocytes secrete IL-17, which stimulates the production of IL-1, IL-6, IL-8, MCP-1, Gro-a, G-CSF, GM-CSF, TNF- $\alpha$, and PGE2. Such mediators can amplify the enlistment of monocytes, neutrophils, and other immune cells in the inflammatory response. All these immune signaling pathways are designed to create an inflammatory environment to eradicate SARS-CoV-2 [1,4]. However, in this short duration of the present pandemic, studies have shown that SARS-CoV-2 has several defence mechanisms, making its eradication more difficult than anticipated [4].

Next, Vitamin D is an essential component of human health and functions in various ways to facilitate optimal health. Vitamin D is either obtained from a healthy diet rich in dairy, fish, and eggs or through conversion from the naturally existing inactive precursor found in the skin [5]. The precursor molecule 7-dehydrocholesterol, located in the upper epidermal cells, undergoes thermal transformation to pre-vitamin D3 by UV B radiation's direct action. This precursor molecule gets converted to Cholecalciferol (inactive Vitamin D3) through a non-enzymatic reaction in the deeper skin layers [6]. Cholecalciferol is later converted to inactive 25hydroxyvitamin D and active 1,25-dihydroxyvitamin D in the liver and kidney by 25-hydroxylase and 1-alpha-hydroxylase, respectively [6]. After binding to its intracellular receptor, active Vitamin D3 acts via alterations in gene expression to increase serum calcium levels by augmenting intestinal calcium absorption and kidney reabsorption of calcium that is filtered and promotes calcium resorption from the bone [6]. While regulating the serum calcium, Vitamin D3 also modulates serum phosphorus levels by increasing phosphorus absorption by intestinal cells [5], [6]. Active Vitamin D3 is also necessary for efficient bone mineralization, which enhances bone density [6]. In individuals with a Vitamin D deficient diet, pathological conditions like Rickets and Osteomalacia occur [5]. Numerous research trials have also shown notable improvement in bone density with reduced fracture risk in long-term Vitamin D supplementation alone in patients with osteoporosis, a condition commonly seen in the elderly characterized by low bone density and increased fracture risk [5].

While Vitamin D's role in calcium homeostasis and bone mineralization is well described in the literature, its functions extend beyond bone health. The Vitamin D receptor expression by several cell types such as pancreatic cells and macrophages highly suggests this $[7,8]$. Also notable is the presence of 1alpha-hydroxylase in other cells, including intestinal, vascular smooth muscle cells, and macrophages, in addition to renal cells, the primary site of active Vitamin D synthesis [7]. Two notable extra-skeletal functions of Vitamin D include cell proliferation with differentiation and modulation of the immune system. In recent literature, Vitamin D is described as a powerful immune modulator [8]. Its role in immunity was first suggested by the use of Cod liver oil, which is rich in Vitamin D, to treat pulmonary tuberculosis during the $1800 \mathrm{~s}$ before the discovery of antibiotics [7, 9]. Many recent studies have further elucidated the role of Vitamin D in immunity and inflammation. Furthermore, it is also proven that Vitamin D supplementation causes upregulation of expression of the antioxidation genes (glutathione reductase and glutamatecysteine ligase modifier subunit) [10].

Given the common link between COVID-19 and Vitamin D as they relate to the immune system, this study aims to conduct a review to explore the role of Vitamin D supplementation in COVID-19 prophylactically and therapeutically.

\section{Vitamin D DEFICIENCY AND COVID-19 - "COVITD19"}

A meta-analysis shows a beneficial effect of daily Vitamin D supplementation when given to vitamin D deficient patients with acute respiratory infections. After this meta-analysis, several other studies show varied results regarding this beneficial effect. During the winter season with the paucity of sunlight and staying at home further prevent sunlight exposure leading to Vitamin D deficiency commonly occurring in such times, thus, it can be considered that Vitamin D supplements should be urged especially in people with high risk for vitamin D deficiency such as obese, elderly, and lack of sunlight exposure [11]. Moreover, vitamin D deficiency and higher mortality rates in COVID-19 patients have been associated in a study in Europe. It is also hinted that mortality rates in COVID-19 are lower in countries nearer to the equator owing to abundant ultraviolet radiations and lesser prevalence of vitamin D deficiency. Another meta-analysis identified 1542 articles and chose 27, which depicted that severe cases of COVID-19 and rate of hospitalization are positively associated with vitamin $\mathrm{D}$ deficiency in comparison to mild cases of COVID-19 [12]. 


\section{Potential Mode of Action OF Vitamin D AGainst SARS COV 2}

SARS-CoV-2 has a genome that is 79\% identical to SARS$\mathrm{CoV}$. Just six $\mathrm{CoV}$ species have been reported with consequences for human health until late December 2019, four of which (229E, OC43, NL63, and HKU1) can induce moderate symptoms like the common cold. SARS-CoV2, along with the other two CoV strains (SARS-CoV and MERS$\mathrm{CoV}$ ), is currently capable of causing mortality. Spike, membrane, nucleocapsid, and envelope are the four major proteins encoded by the CoV genome [13]. Vitamin D is a central regulator of the renin-angiotensin pathway, which SARS-CoV-2 uses to gain access to host cells. Furthermore, vitamin D modulates various immune system pathways to contain the virus, including dampening SARS-CoV-2 entry and replication, reducing pro-inflammatory cytokines, and increasing anti-inflammatory cytokines, increasing the synthesis of a natural antimicrobial peptide, and activating protective cells such as macrophages that can kill SARS-CoV2 [14]. COVID-19 pathology is the product of a complex relationship between SARS-CoV2 and the body's immune system. Calcitriol (1,25-dihydroxyvitamin D3) has a strong effect on the ACE2/Ang(1-7)/MasR axis, resulting in increased ACE2 expression. SARS-CoV-2 infection is mediated by the ACE2 receptor on the host cell [15]. The virus's spike protein is responsible for viral entry into host cells through sensing and binding to the ACE2 receptor, which is strongly expressed in a variety of cells, including type II alveolar and myocardial cells, as well as the kidney's proximal tubule cells [13]. Vitamin D's function in modulating the innate and adaptive immune systems is not well established, but it is significant, and vitamin D deficiency is linked to an increased vulnerability to respiratory infections [16]. Vitamin D strengthens innate cellular immunity by inducing antimicrobial peptides such as the human cathelicidin LL-37, as well as 1,25dihydroxyvitamin $\mathrm{D}$ and defensins, all while preserving close junctions, gap junctions, and adherens junctions. Furthermore, the 1,25(OH)2D-vitamin D receptor complex enhances cathelicidin gene transcription by acting on the cathelicidin gene promoter vitamin D reaction components [17]. In early 2020, Alipio published a retrospective analysis containing data from 212 COVID-19 study participants, which found an important connection between vitamin D deficiency and poor health outcomes in these cases. He also mentioned that the most serious COVID-19 patients had the highest level of deficiency. Furthermore, the report found that the chances of having a moderate clinical outcome rather than a serious outcome increased by 7.94 times with each increment in the standard deviation of $25(\mathrm{OH}) \mathrm{D}$, while the odds of having a mild clinical outcome rather than a critical outcome increased by 19.61 times [15]. Maghbooli et al. [18] published another important study that found a connection between serum 25(OH)D levels and clinical outcomes in 235 patients with COVID-19 infection. After controlling for conflicting factors, researchers discovered an important link between 25(OH)D levels and disease incidence, patient mortality, CRP levels, and increased lymphocyte percentage. Furthermore, adequate vitamin D levels were linked to a lower risk of unconsciousness and hypoxia. Furthermore, only $9.7 \%$ of patients who were vitamin $\mathrm{D}$ deficient died, while the mortality rate for those with serum $25(\mathrm{OH}) \mathrm{D}$ levels less than $30 \mathrm{ng} / \mathrm{ml}$ was as high as $20 \%$. As a result, lower CRP levels and a higher percentage of lymphocytes in circulation suggest that vitamin D deficiency can play a role in modulating the immune response of infected patients [15], [18]. Vitamin D deficiency affects over half of the world's population. Since hypovitaminosis D is an independent risk factor for total mortality in the general population, its high prevalence is a major public health concern [13]. Vitamin D deficiency may have a wide range of effects. It affects the growth and progression of chronic diseases including heart disease, bone health, autoimmunity, type 2 diabetes, cancer, and depression [13]. Deficiency impairs macrophage maturation, production of macrophage-specific surface antigens, production of the lysosomal enzyme acid phosphatase, and secretion of $\mathrm{H}_{2} \mathrm{O}_{2}$, both of which are important functions in their antimicrobial role [16]. A low serum concentration of $25(\mathrm{OH}) \mathrm{D}$ is a risk factor for acute respiratory tract infection, according to a meta-analysis of 14 retrospective trials $(\mathrm{OR}=1.83$; 95\% CI, 1.42-2.37; P-value for heterogeneity, 0.001). Vitamin D supplementation, on the other hand, is linked to a reduced risk of acute respiratory tract infections, according to a meta-analysis of 25 randomized clinical trials ( $\mathrm{OR}=0.88 ; 95 \% \mathrm{CI}, 0.81-0.96 ; \mathrm{P}=0.003$; $\mathrm{P}$-value for heterogeneity, 0.001) [13]. COVID-19 patients may experience neurological symptoms such as loss of taste and scent, headaches, and so on. Because of the extremely infectious virus, few autopsies of these cases have been performed, little research has been done on how SARS-CoV-2 affects the nervous system. Previous data from SARS-CoV studies have shown the existence of SARS-CoV in neurons of SARS patients' brains, implying that the coronavirus can attack the nervous system through the olfactory bulb. Vitamin D's neuroprotective properties are linked to the modulation of neurotrophins, which are essential for nerve cell survival, differentiation, and maintenance in both the peripheral and central nervous systems. In neurons, glial cells, and Schwann cells, vitamin D induces the release of NGF, brain-derived neurotrophic factor (BDNF), neurotrophin-3 (NT3), glial neurotrophic factor, and the neurotrophin receptor p75NTR. Vitamin D supplementation has also been shown in clinical trials to help reduce acute respiratory infections by modulating the innate immune response and increasing antibody synthesis following vaccination [19].

\section{Vitamin D AS A PROPHYLACTIC}

\section{A. Does Vitamin D Deficiency Leave Us Vulnerable to Viral Infections?}

Vitamin D deficiency could be a vital component in susceptibility to viral infections; to better understand this, we must understand the role Vitamin D plays in regards to immunity. Let's look at the immune response; it consists of two different types of immunity. Adaptive immunity, which is 
mainly mediated by $\mathrm{B}$ and $\mathrm{T}$ lymphocytes, each of which confers its type of immunity (humoral and cell-mediated immunity respectively) and innate immunity, which is the body's primary response to invasion by a pathogen, although this response is non-specific and short-lasting.

\section{B. Vitamin D and Adaptive Immunity:}

The signature cells of cell-mediated immunity, the $\mathrm{T}$ lymphocytes, further divide amongst themselves to form subsets. The CD4+ subset of $\mathrm{T}$ cells further differentiates into Th1 cells, a subgroup of helper $\mathrm{T}$ cells that are responsible for the production of cytokines such as IL-2, TNF-alpha, and IFNgamma and it is this particular response that is integral to the defense against viral infections [20]. Vitamin D modulates Th cell proliferation and promotes suppressor T cells, resulting in a limited inflammatory response [20]. It does this by shifting the Th1 phenotype to the Th2 phenotype so that it will not differentiate into the Th17 phenotype, which is more inflammatory. COVID-19 elicits an exaggerated immune response (termed as 'cytokine storm') which manifests as critical lung injury and multiorgan failure which leads to death. Vitamin D regulated Th cell proliferation could effectively prevent or diminish this response [21]. Similarly, Vitamin D also inhibits the proliferation of B lymphocytes and is proapoptotic [6], [20].

\section{Vitamin D and Innate Immunity}

If $\mathrm{B}$ and $\mathrm{T}$ lymphocytes are the signature cells of adaptive immunity, then pathogen recognition receptors are the molecules that play a signature role in innate immunity. Tolllike receptors (TLRs) are a type of pathogen recognition receptor and are present in the cells that carry out the primary response of innate immunity, that is, monocytes, macrophages, neutrophils, dendritic cells, and epithelial cells [20]. TLRs function by recognizing molecules that are specific to pathogens, causing them to become activated and release cytokines that induce reactive oxygen species, antimicrobial peptides, cathelicidins and defensins. Increased Vitamin D alpha-hydroxylase and Vitamin D receptor expression in monocytes, macrophages and epidermal keratinocytes inhibit TLRs [6], [20]. Although Vitamin D alpha-hydroxylase mainly functions in the liver and kidneys, it can be found in extrarenal tissues as well and PRRs, including TLRs, may be able to activate this enzyme in those tissues. Vitamin D also acts on other cells such as monocytes and dendritic cells to prevent the release of pro-inflammatory cytokines [20], [22]. Vitamin D's overall effect on innate immunity likely is to cause shifts and changes to prevent the release of damaging cytokines from first-line defence cells by blocking PRR signaling and inhibiting transcription of intranuclear and intracytoplasmic receptor ligands to block the effects of TLRs [6], [20], [21].

\section{Clinical Trials}

Considering the immunomodulatory and protective role of Vitamin D against viral infection, many studies were conducted in the past to analyse the role of Vitamin D supplementation in the prevention of acute respiratory infection (Influenza/ Upper Respiratory tract infection/ Pneumonia). Though being a type of Acute Respiratory Infection (ARI), there is a need for more specific high-quality data to demonstrate the efficacy of vitamin D supplementation in the prevention of COVID-19. There is a limited number of studies conducted to analyse the relation between vitamin D supplementation and COVID-19 cases. Here are some of those studies:

As demonstrated by Li et al. [22], a retrospective study was conducted using the complete data of COVID-19 cases and related deaths between Jan 22, 2020, to May 23, 2020, across the US. The study population included 48 states plus the District of Columbia on the mainland of the US (the range of latitude covering from N28 to N48), excluding Alaska, Hawaii and other territories that are away from the mainland and with significantly different latitudes. The study included a total of 1,609,488 cases and 91,094 COVID-19 related deaths for analysis. The study aimed to analyze the impact of sunlight and Vitamin D on COVID-19 related cases and deaths, using latitude across different states of the US as an indirect indicator. This analysis was conducted using linear regression and Pearson correlation, after adjusting the population and COVID19 testing rates across the states. The analyses showed that the latitudes were marginally associated with cases $(\mathrm{p}=0.0792)$ and deaths $(p=0.0599)$, with an increase of 2491 cases and 189 deaths of the total numbers in the mainland of the US for every unit of increase of the latitude. For a better understanding of the association between different latitudes and COVID-19 cases and mortality rates, the states were divided into two categories as High Altitude state group (H_LT) (N >37 degrees) and Low Altitude state group(L_LT) (N <37 degrees), considering $\mathrm{N}$ 37degree as a cut-off value. The analysis demonstrated that the rate of COVID-19 cases and related deaths are significantly higher in High altitude study groups (702 cases/100 K population and 43 deaths/100K population respectively) compared to Low altitude study groups (255 cases/100 K population and 11 deaths/100K population respectively) $(\mathrm{p}<0.001)$ [22]. This theory was reinforced by the fact that the top states with COVID-19 cases, more than 90000 or mortality $>5000$, fall under the High altitude study groups (New York, New Jersey, Massachusetts, Michigan, Pennsylvania) or just close to the N37 latitude line (California). Latitude plays a significant role in the level of the body's Vitamin D synthesis from the sun, which is best explained by the fact that people residing in the areas above 37 degrees north or below 37 degrees south of the equator make very little to no vitamin D synthesis from the sun. This study used latitude as an indicator of sunlight and Vitamin D level that is the natural source of Vitamin D and its influence on COVID-19 cases and mortalities [22]. However, it is also influenced by many other factors like clothing, sunscreen use, skin pigmentation, dietary supplement, age, obesity etc. These findings warrant the need for more randomised controlled trials, cohorts, and clinical studies to assess the role of vitamin D supplementation in the prevention of COVID-19. Finally, this study exhibited a potential relationship between latitude, an indicator of sunlight 
and Vitamin-D status and COVID-19 cases and related mortalities [22].

As demonstrated by Kun et al. [23], a case-control study was conducted using data of COVID-19 patients admitted from February 16th, 2020, to March 16th, 2020 at Yongwu Hospital of the people's hospital of Guangxi Zhuang Autonomous Region, Nanning, China and the healthy control group from the Physical Examination Center of the same institution. The confounding factors including age, sex and comorbidities were matched between the two groups. The study population consisted of 62 COVID-19 confirmed cases and 80 healthy controls. The main aim of this study was to evaluate the potential impact of Vitamin D on COVID-19 incidence, its prognosis and clinical presentation among the Chinese population. The Statistical analysis of the study was conducted using a multivariate logistic regression model to assess the relation between Vitamin D status and incidence/severity of COVID-19. The study estimated that serum 25hydroxyvitamin-D levels were statistically low in COVID19 patients $(55.6 \mathrm{nmol} / \mathrm{L})$, compared to that of healthy controls $(71.8 \mathrm{nmol} / \mathrm{L})$. Furthermore, they found that the rate of Vitamin D deficiency was higher in the case group $(n=26[41.9 \%])$, compared to that in the control group $(n=$ $15[18.8 \%])(\mathrm{OR}=3.13,95 \% \mathrm{CI}=1.47-6.66)$ [23]. Also, there was a statistically significant association between Vitamin D deficiency and severe/critical disease $(\mathrm{OR}=15.18,95 \% \mathrm{CI}=$ 1.23-187.45) after adjusting the possible confounders. The study also showed that the severe/critical cases are usually elderly (median $=65)$, likely to exhibit clinical symptoms (fever, cough, diarrhoea) and have underlying medical comorbidities (diabetes, Hypertension, Renal failure) compared to mild/moderate cases [23]. In conclusion, this evidence-based study demonstrated the association of Vitamin D status on the incidence and prognosis of COVID-19, thus signifying the prophylactic use of Vitamin D supplementation in preventing COVID19 and also lowering the potential adverse events associated with the disease. A daily effective dose of Vitamin D3 is advisable to maintain the optimal concentration $>50 \mathrm{nmol} / \mathrm{L}$ in such individuals. Although this study shows a strong association between vitamin D deficiency and COVID19 incidence and severity, the statistical power is lower compared to standard epidemiological studies because of the small population size. This warrants the need for studies with a large sample size and better representativeness [23].

Avolio and Acataneo et al. [24] conducted a retrospective cohort study at Canton of Tessin, Switzerland using the data of patients who underwent a nasopharyngeal PCR analysis for COVID-19 and serum 25hydroxyvitamin-D measurements between 1st March 2020 and 14th April 2020. Meanwhile, patients who underwent 25 hydroxyvitaminD measurement during the same period (1 March to 14 April) of 2019 were considered as a control group for comparison. A total of 107 patients who presented with acute respiratory symptoms (e.g., cough, breathing difficulties, sore throat) underwent PCR analysis during this period, among which 27 were SARS-CoV2 positive and 80 were negative. Furthermore, a total of 1377 patients had got 25hydroxyvitamin D level measured in 2019 (control group) [24]. The objective of the study was to evaluate the association of vitamin D status with the risk of acquiring COVID-19 infection in particular, although there is strong evidence to justify the protective role of vitamin D against other acute respiratory infections. The Shapiro-Wilk test, Mann-Whitney/ Kruskal-Wallis test and Spearman's rank correlation were adopted to analyse various variables in this study. At the end of the study, it was estimated that patients who tested positive for SARS-CoV-2 PCR had statistically significant $(\mathrm{p}=0.004)$ lower 25hydroxyvitamin-D levels (11.1 $\mathrm{ng} / \mathrm{mL})$ compared to those tested negative $(24.6 \mathrm{ng} / \mathrm{mL})$ [24]. Besides, an even stronger statistically significant $(p<0.001)$ difference was noted in 25hydroxyvitamin-D levels between SARS-CoV-2 positive patients of 2020 and patients of 2019(24.6 ng/mL). On stratification of 2019 and 2020 positive patients by gender, the study demonstrated statistically significance difference: $25.6 \mathrm{ng} / \mathrm{mL}$ vs $9.3 \mathrm{ng} / \mathrm{mL}(\mathrm{p}=0.019)$ in women and $22.9 \mathrm{ng} / \mathrm{mL}$ vs. $11.4 \mathrm{ng} / \mathrm{mL}(\mathrm{p}=0.005)$ in men respectively [24]. Similarly, when the study group was stratified by age ( $0-70$ years and $>70$ years), the statistically significant difference $(\mathrm{p}=0.037)$ in 25 hydroxyvitamin $\mathrm{D}$ level was observed between the 2020 SARS-Co-V positive $(9.3 \mathrm{ng} / \mathrm{mL})$ and negative patients $(23.1 \mathrm{ng} / \mathrm{mL})$ only in the category of $>70$ years age group but not in $<70$ years age group. Also, a stronger difference $(\mathrm{p}<0.001)$ was noted when comparing 2019 patients $(26.4 \mathrm{ng} / \mathrm{mL})$ and 2020 SARS-CoV2 positive $(9.3 \mathrm{ng} / \mathrm{mL})$ patients in the $>70$ years group. In conclusion, this cohort study accomplished the significance of Vitamin D supplementation in preventing the incidence and progression of COVID-19 infection, along with estimating the higher incidence in the elderly group ( $>70$ years). These findings were further justified by other authors, evidenced by the higher rate of COVID-19 incidence in winter, and in elderly and patients with comorbidities, all of which contribute to lower 25 hydroxyvitamin D levels. This signifies the importance of daily vitamin-D supplementation to achieve a serum level of more than $40-60 \mathrm{ng} / \mathrm{mL}$ especially in the susceptible population, and also the need for large scale and randomised control studies [24].

\section{Vitamin D AS A Therapeutic}

Vitamin D is a fat-soluble vitamin present in the Beef liver, cheese, and egg yolks as Vitamin D3 and its metabolite $25(\mathrm{OH}) \mathrm{D} 3$. Vitamin D synthesis is triggered when UV rays strike the skin, and it is an essential element for maintaining a healthy mineral and bone metabolism [25].

In Vitro, studies have found potent antimicrobial and antiinflammatory effects [26] of Vitamin D, and in the animal models, vitamin $\mathrm{D}$ administration attenuates acute organ dysfunction, including acute lung injury [27]. Patients with lower levels of Vitamin D are associated with poor clinical outcomes including the COVID-19 patients [28].

Grant et al. [29] recommended taking 10,000 IU/day of vitamin $\mathrm{D}_{3}$ for a few weeks to rapidly raise $25(\mathrm{OH}) \mathrm{D}$ concentrations, followed by $5000 \mathrm{IU} / \mathrm{d}$. The goal should be to raise $25(\mathrm{OH}) \mathrm{D}$ concentrations above $40-60 \mathrm{ng} / \mathrm{mL}$ (100- 
$150 \mathrm{nmol} / \mathrm{L}$ ), to reduce the risk of influenza and/or COVID-19. Higher doses are advised for the infected patients.

Short term, high-dose vitamin D supplementation for COVID-19 disease: a randomised, placebo-controlled, study (SHADE study) measured the effect of high dose, oral cholecalciferol on SARS-CoV-2 viral clearance in asymptomatic or mildly symptomatic COVID-19 positive, vitamin $\mathrm{D}$ deficient i.e., $25(\mathrm{OH}) \mathrm{D}<20 \mathrm{ng} / \mathrm{ml}$ patients. They found that the greater proportion of vitamin D-deficient individuals with SARS-CoV-2 infection turned SARS-CoV-2 RNA negative with a significant decrease in fibrinogen on high-dose cholecalciferol supplementation [30]. This study is further discussed in detail later in this review.

However, the multicenter, double-blind, randomized, placebo-controlled trial conducted in Sao Paulo, Brazil did not find any significant reduction in the length of hospital stay in these patients. They investigated the effect of a single high dose of vitamin D3 on hospital length of stay in COVID-19 patients and gave a single oral dose of $200000 \mathrm{IU}$ of vitamin D3 ( $\mathrm{n}=$ $120)$ or placebo $(\mathrm{n}=120)$ [31].

Meltzer et al. [32] analyzed the link between vitamin D deficiency, its treatment, and the association between the incidence of COVID-19, using data from the electronic health record at the University of Chicago Medicine. They compared the patients with a low measured basal level of vitamin D and no supplementation treatment versus patients with a low basal level of vitamin D but supplemented with this vitamin. In the results, they found that the non-supplemented group has a significantly higher number of positive tests for COVID-19. Among the treated patients, the vitamin D protective effect against the SAR-CoV-2 virus infection was significant only in the group with basal vitamin D deficiency [32].

Another systematic review studied the link between vitamin D status and COVID-19 infection and prognosis and found that seven $(77.8 \%)$ studies have a significant correlation between these parameters. They concluded that blood vitamin D status can determine the risk of infection, severity, and mortality from COVID-19. Therefore, it is suggested to maintain appropriate levels of Vitamin D through supplementation or natural methods, e.g., sunlight on the skin [33].

\section{Natural AND (VS) Supplemental Vitamin D}

The principal supply of vitamin $\mathrm{D}$ is cutaneous production from ultraviolet-B rays which accounts for almost $90 \%$. The Cholesterol-like precursor (7-dehydrocholesterol) is converted by skin to pre-vitamin $\mathrm{D}$, which isomerizes into vitamin D3. Vitamin D3 (Cholecalciferol) is exclusively an animal source vitamin, whereas vitamin D2 (ergocalciferol) is a plant source vitamin. Both of them are inactive forms of vitamin D. Some examples of natural sources of these inactive forms include oily fishes like sardines, herring, tuna, mackerel, and salmon, egg yolks, liver, or organ meats. Thus, humans can receive vitamin D from ultraviolet radiation, vitamin D-rich diets, fortified diets (breakfast cereals), and supplemental vitamin D3 and D2. Both vitamin D3 and D2 are converted to active forms by the liver and kidney. Firstly, these inactive forms undergo 25- hydroxylation in the liver to form 25(OH)D (calcidiol). Then it undergoes 1-alpha-hydroxylation in kidneys to form the active form of vitamin D which is $1,25(\mathrm{OH}) 2 \mathrm{D}$ (calcitriol). There are also some extra-renal locations where 1-alpha-hydroxylation occurs, these sites include alveolar macrophages, osteoblasts, lymph nodes, placenta, colon, breasts, and keratinocytes [34], [35]. It is also noted in a meta-analysis and systematic review that vitamin D3 supplementation is more effective in increasing serum level of 25(OH)D than vitamin D2 supplementation. The same effect was seen even after changing dosage or route of administration (oral vs intramuscular) for both types of supplements [35].

\section{DOSING, DURATION OF THERAPY/PROPHYLAXIS AND ADVERSE EFFECTS OF VITAMIN D}

Severe COVID-19 related complications and mortality can be prevented by preventing a cytokine storm which is caused by suppression of the adaptive immune system. It is a known fact that Vitamin D does immune regulation by enhancing the innate immune system which further suppresses the adaptive immune system and prevents release of cytokines [6]. A study evaluated the intensity of cytokine storm in severe COVID-19 using C-reactive protein (CRP) levels and potential impact of Vitamin D on CRP levels and concluded that there is a potential for a $15.6 \%$ reduction in the risk of severe COVID-19 cases by Vit D [36].

A study suggested that patients with severe vitamin D deficiency experienced the maximum benefits from the supplementation, and the protective role is highest in patients with baseline serum 25(OH)D levels $<25 \mathrm{nmol} / \mathrm{L}$ compared to those with serum $25(\mathrm{OH}) \mathrm{D}$ concentrations $>25 \mathrm{nmol} / \mathrm{L}$. It also concluded that Vitamin D supplementation reduces the risk of acute respiratory tract infections [37]. Another study concluded that people at risk of vitamin D deficiency should be supplemented to maintain circulating 25(OH)D levels between $75-125 \mathrm{nmol} / \mathrm{L}$, especially during the global pandemics [28].

As mentioned earlier, prophylactically, people at the risk of influenza and/or COVID-19 should be given 10,000 IU/d of vitamin D3 for a few weeks to rapidly raise 25(OH)D concentrations, followed by $5000 \mathrm{IU} / \mathrm{d}$ to maintain levels of 25(OH)D3 above 40-60 $\mathrm{ng} / \mathrm{mL}(100-150 \mathrm{nmol} / \mathrm{L})$ [29]. Although, there is no scientific consensus about the optimal dose of Vitamin D for COVID-19 patients currently, the Institute of Medicine recommended a dietary allowance, or RDA, for vitamin D at 600 international units (IU) per day for young adults and $800 \mathrm{IU}$ per day for adults older than 70 years [38]. In another study, Wimalawansa et al. suggested a loading dose of 200,000-300,000 IU in 50,000-IU capsules reduces the risk and severity of COVID-19 [39].

A randomized clinical trial was done to study the effect of high dose, oral cholecalciferol supplementation on SARSCoV-2 viral clearance in asymptomatic or mildly symptomatic SARS-CoV-2 RNA positive vitamin D deficient individuals in a tertiary care hospital in north India. 40 participants were randomized into an intervention group $(\mathrm{n}=16)$ and control arm $(n=24)$. The primary endpoint was the proportion of patients 
with SARS CoV-2 RNA negative before day-21. The intervention group was given daily 60,000 IU of cholecalciferol for 7 days. If the target serum levels of 25hydroxy Vitamin D (>50 ng/ml) are not achieved at day 7 , then they are continued with the same until 14 days. The rest of them were supplemented with weekly doses until day 14. 12 participants from the intervention arm reached target levels by day $14.62 .5 \%$ of participants from the intervention group and $20.8 \%$ of participants from the control group became SARSCoV-2 RNA negative before day 21. Fibrinogen levels were significantly decreased in the intervention group. It can be concluded that Vitamin D can be useful in the treatment of asymptomatic COVID-19 positive, Vitamin D deficient individuals; thus, reducing transmission [30].

A randomized clinical study was done in a university hospital setting in Spain to evaluate the effect of calcifediol (the main metabolite of Vitamin D3) treatment on Intensive Care Unit Admission and Mortality rate among Spanish patients hospitalized for COVID-19. The primary endpoint was the rate of ICU admission and deaths. Seventy-six patients were randomized into an intervention group $(n=50)$ and control group $(n=26)$, with the intervention group receiving 2 calcifediol $(0.532 \mathrm{mg})$ on day 1 and 1 calcifediol $(0.266 \mathrm{mg})$ on day 3 and day 7 and then weekly until discharge or ICU admission; and a control group receiving no calcifediol treatment. Of the patients treated with calcifediol, none died, and all were discharged, without complications. The 13 patients not treated with calcifediol, who were not admitted to the ICU, were discharged. Of the 13 patients admitted to the ICU, two died and the remaining 11 were discharged. It can be concluded that high dose calcifediol treatment can reduce the risk of ICU admissions in COVID patients and thus helps in reducing the severity of the disease [40].

A quasi-experimental study was done to determine whether bolus vitamin D supplementation taken either regularly over the preceding year or after the diagnosis of COVID-19 was effective in improving survival among 77 hospitalized frail elderly COVID-19 patients. The study was done on participants who were regularly supplemented with oral bolus doses of Vitamin D3 in the preceding year (Group 1), who received an oral supplement of 80,000 IU vitamin D3 within a few hours of the diagnosis of COVID-19 (Group 2) and a comparator group who did not receive any of these. The primary outcome was 14-day mortality, and the secondary outcome was the highest(worst) clinical score for improvement (OSCI Score). Although 93.1\% of Group 1, 81.2\% of Group 2 and $68.7 \%$ of Group 3 showed a 14-day survival rate, there was no significant difference between Group 2 and Group 3. Group 1 also showed lower OSCI scores. Therefore, it was concluded that regular bolus doses of Vitamin D supplementation given prophylactically were useful in decreasing the severity of COVID-19 and in improving survival rate [41].

\section{ADVERSE EFFECTS}

According to the U.S. Institute of Medicine, daily doses of Vit D supplementations $<10,000$ IU/day with no adverse effects, the value for the upper limit (UL) was corrected to 4000 IU/day based on all-cause mortality and chronic disease outcomes [38].

Some health groups recommend taking from 800 to $2,000 \mathrm{IU}$ per day, but some clinicians recommend higher doses such as a 5,000 IU OTC capsule or even 10,000 IU per day without symptoms of toxicity [42]. However, Randomized Controlled Trials have found that 3 -year-treatment of vitamin $\mathrm{D}$ at a dose of 10,000 IU/d can lower radial bone and tibial bone mineral density [43].

Martineau et al. confirmed the safety and the protective effect against acute respiratory tract infection of regular oral vitamin D2/D3 intake (up to $2000 \mathrm{IU} / \mathrm{d}$ without an additional bolus), especially in subjects with vitamin D deficiency [37].

\section{CONCLUSION}

This review aimed at determining the role of Vitamin D as a prophylactic and therapeutic agent in COVID-19 infection. Although the direct evidence is scarce, there are indications of Vitamin D being a potent immunomodulator and protective against acute viral respiratory tract infections. Additionally, in many individuals, low Vitamin D levels seem to be associated with increased incidence and poor prognosis of COVID-19. From our literature review, we conclude that there are more advantages over adverse effects of using Vitamin D as a prophylactic and a therapeutic in the management of COVID19. Many studies have been conducted which proved that over time Vitamin D did show improvement in the survival rate. However, further clinical trials are needed to assess the direct impact to minimize the COVID-19 infections.

\section{ACKNOWLEDGMENT}

A very special thanks to Dr. Marcos Sanchez-Gonzales and Dr. Jack Michel for their tremendous support and Team 11 initiative. Thanks to Larkin Health System!

\section{REFERENCES}

[1] Chowdhury, M. A., Hossain, N., Kashem, M. A., Shahid, Md. A., \& Alam, A. Immune response in COVID-19: A review. Journal of Infection and Public Health. 2020; 13(11), 1619-1629. https://doi.org/10.1016/j.jiph.2020.07.001.

[2] Zheng YY, Ma YT, Zhang JY, et al. COVID-19 and the cardiovascular system. Nature Reviews Cardiology. 2020; 17: 259-260.

[3] Yazdanpanah, F., Hamblin, M. R., \& Rezaei, N. The immune system and COVID-19: Friend or foe? Life Sciences. 2020; 256, 117900. https://doi.org/10.1016/j.1fs.2020.117900.

[4] Paces, J., Strizova, Z., Smrz, D., \& Cerny, J. COVID-19 and the Immune System. Physiological Research. 2020; 379-388. https://doi.org/10.33549/physiolres.934492.

[5] Laird, E., Ward, M., McSorley, E., Strain, J. J., \& Wallace, J. Vitamin D and Bone Health; Potential Mechanisms. Nutrients. 2010; 2(7), 693-724. https://doi.org/10.3390/nu2070693.

[6] Aranow C. Vitamin D and the immune system. Journal of investigative medicine: the official publication of the American Federation for Clinical Research. $2011 ; \quad 59(6), \quad 881-886$. https://doi.org/10.2310/JIM.0b013e31821b8755.

[7] Hewison, M., Burke, F., Evans, K. N., Lammas, D. A., Sansom, D. M., Liu, P., Modlin, R. L., \& Adams, J. S. Extra-renal 25-hydroxyvitamin 
D3-1 $\alpha$-hydroxylase in human health and disease. The Journal of Steroid Biochemistry and Molecular Biology. 2007; 103(3-5), 316-321. https://doi.org/10.1016/j.jsbmb.2006.12.078.

[8] Lai, Y.-H., \& Fang, T.-C. The Pleiotropic Effect of Vitamin D. ISRN Nephrology. 2013; 1-6. https://doi.org/10.5402/2013/898125.

[9] Prietl, B., Treiber, G., Pieber, T. R., \& Amrein, K. Vitamin D and immune function. Nutrients. 2020; 5(7), 2502-2521. https://doi.org/10.3390/nu5072502.

[10] Grant, W. B., Lahore, H., McDonnell, S. L., Baggerly, C. A., French, C. B., Aliano, J. L., \& Bhattoa, H. P. Evidence that Vitamin D Supplementation Could Reduce Risk of Influenza and COVID-19 Infections and Deaths. Nutrients. 2020; 12(4), 988. https://doi.org/10.3390/nu12040988.

[11] Zemb P, Bergman P, Camargo CA Jr, Cavalier E, Cormier C, Courbebaisse M, Hollis B, Joulia F, Minisola S, Pilz S, Pludowski P, Schmitt F, Zdrenghea M, Souberbielle JC. Vitamin D deficiency and the COVID-19 pandemic. J Glob Antimicrob Resist. 2020 Sep;22:133-134. doi: 10.1016/j.jgar.2020.05.006. Epub 2020 May 29. PMID: 32474141; PMCID: PMC7256612.

[12] Pereira M, Dantas Damascena A, Galvão Azevedo LM, de Almeida Oliveira T, da Mota Santana J. Vitamin D deficiency aggravates COVID19: systematic review and meta-analysis. Crit Rev Food Sci Nutr. 2020 Nov 4:1-9. doi: 10.1080/10408398.2020.1841090. Epub ahead of print. PMID: 33146028.

[13] Turrubiates-Hernández FJ, Sánchez-Zuno GA, González-Estevez G, Hernández-Bello J, Macedo-Ojeda G, Muñoz-Valle JF. Potential immunomodulatory effects of vitamin $\mathrm{D}$ in the prevention of severe coronavirus disease 2019: An ally for Latin America (Review). Int J Mol Med. 2021;47(4):1.doi: 10.3892/ijmm.2021.4865.

[14] Kumar R, Rathi H, Haq A, Wimalawansa SJ, Sharma A. Putative roles of vitamin $\mathrm{D}$ in modulating immune response and immunopathology associated with COVID-19. Virus Res. 2021;292:198235. doi:10.1016/j.virusres.2020.198235.

[15] Ilie PC, Stefanescu S, Smith L. The role of vitamin D in the prevention of coronavirus disease 2019 infection and mortality. Aging Clin Exp Res. 2020;32(7):1195-1198. doi:10.1007/s40520-020-01570-8.

[16] Santaolalla A, Beckmann K, Kibaru J, Josephs D, Van Hemelrijck M, Irshad S. Association Between Vitamin D and Novel SARS-CoV-2 Respiratory Dysfunction - A Scoping Review of Current Evidence and Its Implication for COVID-19 Pandemic. Front Physiol. 2020;11:564387. Published 2020 Nov 26. doi:10.3389/fphys.2020.564387.

[17] Murdaca, G., Pioggia, G. \& Negrini, S. Vitamin D and Covid-19: an update on evidence and potential therapeutic implications. Clin Mol Allergy 18, 23 (2020). https://doi.org/10.1186/s12948-020-00139-0.

[18] Maghbooli Z, Sahraian MA, Ebrahimi M, Pazoki M, Kafan S, et al. (2020) Vitamin D sufficiency, a serum 25-hydroxyvitamin D at least 30 $\mathrm{ng} / \mathrm{mL}$ reduced risk for adverse clinical outcomes in patients with COVID-19 infection. PLOS ONE 15(9): e0239799. https://doi.org/10.1371/journal.pone.0239799.

[19] Xu, Y., Baylink, D.J., Chen, CS. et al. The importance of vitamin d metabolism as a potential prophylactic, immunoregulatory and neuroprotective treatment for COVID-19. J Transl Med 18, 322 (2020). https://doi.org/10.1186/s12967-020-02488-5.

[20] Gruber-Bzura BM. Vitamin D and Influenza-Prevention or Therapy?. Int $J$ Mol Sci. 2018;19(8):2419. Published 2018 Aug 16. doi:10.3390/ijms19082419.

[21] Cutolo M, Paolino S, Smith VEvidences for a protective role of vitamin D in COVID-19RMD Open 2020;6:e001454. doi: 10.1136/rmdopen2020-001454.

[22] Li Y, Li Q, Zhang N, Liu Z. Sunlight and vitamin D in the prevention of coronavirus disease (COVID-19) infection and mortality in the United States (preprint, in review); 2020. doi:10.21203/rs.3.rs-32499/v1.

[23] Kun Ye, Fen Tang, Xin Liao, Benjamin A. Shaw, Meiqiu Deng, Guangyi Huang, Zhiqiang Qin, Xiaomei Peng, Hewei Xiao, Chunxia Chen, Xiaochun Liu, Leping Ning, Bangqin Wang, Ningning Tang, Min Li, Fan $\mathrm{Xu}$, Shao Lin \& Jianrong Yang (2020) Does Serum Vitamin D Level Affect COVID-19 Infection and Its Severity?-A Case-Control Study, Journal of the American College of Nutrition, DOI: 10.1080/07315724.2020.1826005.

[24] D'Avolio A, Avataneo V, Manca A, Cusato J, De Nicolò A, Lucchini R, Keller F, Cantù M. 25-Hydroxyvitamin D Concentrations Are Lower in Patients with Positive PCR for SARS-CoV-2. Nutrients. 2020; 12(5):1359. https://doi.org/10.3390/nu12051359.
[25] Urena-Torres P, Souberbielle JC. Pharmacologic role of vitamin D natural products. Curr Vasc Pharmacol. 2014 Mar;12(2):278-85. doi: 10.2174/15701611113119990020. PMID: 23713872.

[26] Liu PT, Stenger S, Li H, et al. Toll-like receptor triggering of a vitamin D-mediated human antimicrobial response. Science. 2006;311(5768):1770-1773. doi:10.1126/science.1123933.

[27] Kong J, Zhu X, Shi Y, et al. VDR attenuates acute lung injury by blocking Ang-2-Tie-2 pathway and renin-angiotensin system. Mol Endocrinol. 2013;27(12):2116-2125. doi:10.1210/me.2013-1146.

[28] Ali N. Role of vitamin D in preventing of COVID-19 infection, progression and severity. J Infect Public Health. 2020;13(10):1373-1380. doi:10.1016/j.jiph.2020.06.021.

[29] Grant WB, Lahore H, McDonnell SL, Baggerly CA, French CB, Aliano JL, Bhattoa HP. Evidence that Vitamin D Supplementation Could Reduce Risk of Influenza and COVID-19 Infections and Deaths. Nutrients. 2020; 12(4):988. https://doi.org/10.3390/nu12040988.

[30] Rastogi A, Bhansali A, Khare N, et al Short term, high-dose vitamin D supplementation for COVID-19 disease: a randomised, placebocontrolled, study (SHADE study)Postgraduate Medical Journal Published Online First: 12 November 2020. doi: 10.1136/postgradmedj2020-139065.

[31] Murai IH, Fernandes AL, Sales LP, Pinto AJ, Goessler KF, Duran CSC, Silva CBR, Franco AS, Macedo MB, Dalmolin HHH, Baggio J, Balbi GGM, Reis BZ, Antonangelo L, Caparbo VF, Gualano B, Pereira RMR. Effect of a Single High Dose of Vitamin D3 on Hospital Length of Stay in Patients with Moderate to Severe COVID-19: A Randomized Clinical Trial. JAMA. 2021 Mar 16;325(11):1053-1060. doi: 10.1001/jama.2020.26848. PMID: 33595634; PMCID: PMC7890452.

[32] Meltzer DO, Best TJ, Zhang H, Vokes T, Arora V, Solway J. Association of Vitamin D Deficiency and Treatment with COVID-19 Incidence. Preprint. medRxiv. 2020;2020.05.08.20095893. Published 2020 May 13. doi:10.1101/2020.05.08.20095893.

[33] Yisak H, Ewunetei A, Kefale B, et al. Effects of Vitamin D on COVID19 Infection and Prognosis: A Systematic Review. Risk Manag Healthc Policy. 2021;14:31-38. Published 2021 Jan 7. doi:10.2147/RMHP.S291584.

[34] Chang SW, Lee HC. Vitamin D and health - The missing vitamin in humans. Pediatr Neonatol. 2019 Jun;60(3):237-244. doi: 10.1016/j.pedneo.2019.04.007. Epub 2019 Apr 17. PMID: 31101452.

[35] Tripkovic L, Lambert H, Hart K, Smith CP, Bucca G, Penson S, Chope G, Hyppönen E, Berry J, Vieth R, Lanham-New S. Comparison of vitamin D2 and vitamin D3 supplementation in raising serum 25hydroxyvitamin D status: a systematic review and meta-analysis. Am J Clin Nutr. 2012 Jun;95(6):1357-64. doi: 10.3945/ajen.111.031070. Epub 2012 May 2. PMID: 22552031; PMCID: PMC3349454.

[36] Daneshkhah, A., Agrawal, V., Eshein, A. et al. Evidence for possible association of vitamin D status with cytokine storm and unregulated inflammation in COVID-19 patients. Aging Clin Exp Res 32, 2141-2158 (2020). https://doi.org/10.1007/s40520-020-01677-y.

[37] Martineau AR, Jolliffe DA, Hooper RL, et al. Vitamin D supplementation to prevent acute respiratory tract infections: systematic review and metaanalysis of individual participant data. BMJ. 2017;356:i6583. doi:10.1136/bmj.i6583.

[38] Ross AC, Manson JE, Abrams SA, et al. The 2011 report on dietary reference intakes for calcium and vitamin D from the Institute of Medicine: what clinicians need to know. J Clin Endocrinol Metab. 2011;96(1):53-58. doi:10.1210/jc.2010-2704.

[39] Wimalawansa S. Global epidemic of coronaviruses--COVID-19: What can we do to minimize risks - EJBPS. 2020;7:432-438.

[40] Entrenas Castillo M, Entrenas Costa LM, Vaquero Barrios JM, et al. "Effect of calcifediol treatment and best available therapy versus best available therapy on intensive care unit admission and mortality among patients hospitalized for COVID-19: A pilot randomized clinical study." The Journal of Steroid Biochemistry and Molecular Biology. 2020;203:105751. doi:10.1016/j.jsbmb.2020.105751.

[41] Annweiler G, Corvaisier M, Gautier J, et al. Vitamin D Supplementation Associated to Better Survival in Hospitalized Frail Elderly COVID-19 Patients: The GERIA-COVID Quasi-Experimental Study. Nutrients. 2020;12(11):3377. doi:10.3390/nu12113377.

[42] Simonson W. Vitamin D dosing considerations in COVID-19. Geriatr Nurs. 2020;41(5):648-649. doi:10.1016/j.gerinurse.2020.08.011.

[43] Burt LA, Billington EO, Rose MS, Raymond DA, Hanley DA, Boyd SK. Effect of High-Dose Vitamin D Supplementation on Volumetric Bone Density and Bone Strength: A Randomized Clinical Trial. JAMA. 2019;322(8):736. doi:10.1001/jama.2019.11889. 